\title{
A nurse-led sexual rehabilitation intervention after radiotherapy for gynecological cancer
}

\author{
R. M. Bakker ${ }^{1}$ J. W. M. Mens ${ }^{2}$ - H. E. de Groot $^{1}$ - C. C. Tuijnman-Raasveld ${ }^{1}$ \\ C. Braat ${ }^{2}$ - W. C. P. Hompus ${ }^{3}$ - J. G. M. Poelman ${ }^{4}$ - M. S. Laman ${ }^{4}$ - L. A. Velema ${ }^{4}$ • \\ C. D. de Kroon ${ }^{1}$ - H. C. van Doorn ${ }^{3}$ - C. L. Creutzberg ${ }^{4}$ - M. M. ter Kuile ${ }^{1}$
}

Received: 3 June 2016 / Accepted: 10 October 2016 /Published online: 27 October 2016

(C) The Author(s) 2016. This article is published with open access at Springerlink.com

\begin{abstract}
Purpose Although vaginal dilator use after combined pelvic radiation therapy and brachytherapy (RT/BT) is recommended to prevent vaginal shortening and stenosis, women fail to use them and experience sexual problems. A nurse-led sexual rehabilitation intervention targeting sexual recovery and vaginal dilatation was developed. Its feasibility was investigated during a prospective, longitudinal, observational pilot study. Methods Four oncology nurses were specifically trained to conduct the intervention. Gynecologic cancer patients treated with RT/BT were assessed using (i) questionnaires on frequency of dilator use (monthly), sexual functioning, and sexual distress (at baseline and 1, 6, and 12 months) and psychological and relational distress (at 1, 6, and 12 months); (ii) semi-structured interviews (between 6 and 12 months); and (iii) consultation recordings (a random selection of $21 \%$ of all consults).

Results Twenty participants were 26-71 years old $($ mean $=40)$. Eight participants discontinued participation after 3 to 9 months. At 6 months after RT, 14 out of 16 (88\%), and at
\end{abstract}

Electronic supplementary material The online version of this article (doi:10.1007/s00520-016-3453-2) contains supplementary material, which is available to authorized users.

R. M. Bakker

R.M.Bakker@lumc.nl

1 Department of Gynecology, Leiden University Medical Center, Zone VRSP, PO Box 9600, 2300 RC Leiden, the Netherlands

2 Department of Radiotherapy, Erasmus MC Cancer Institute, Rotterdam, the Netherlands

3 Department of Gynecology, Erasmus MC Cancer Institute, Rotterdam, the Netherlands

4 Department of Radiation Oncology, Leiden University Medical Center, Leiden, the Netherlands
12 months 9 out of 12 (75\%), participants dilated regularly, either by having sexual intercourse or by using dilators. Sexual functioning improved between 1 and 6 months after RT, with further improvement at 12 months. Most participants reported that the intervention was helpful and the nurses reported having sufficient expertise and counseling skills.

Conclusions According to the pilot results, the intervention was feasible and promising for sexual rehabilitation and regular dilator use after RT. Its (cost-)effectiveness will be investigated in a randomized controlled trial.

Keywords Gynecological cancer · Pelvic radiation therapy and brachytherapy $\cdot$ Sexual rehabilitation $\cdot$ Dilator use compliance $\cdot$ Nurse-led intervention

\section{Introduction}

About $35 \%$ of gynecologic cancer survivors (GCSs) are treated with primary or postsurgical pelvic radiotherapy (RT) [1]. Patients with more advanced cervical cancer (CC) and vaginal cancer are treated with primary chemoradiotherapy, consisting of pelvic external beam radiation and intrauterine brachytherapy (EBRT/BT), with concurrent cisplatin-based chemotherapy, most often 5-6 weekly infusions of cisplatin $40 \mathrm{mg} / \mathrm{m}^{2}$ [2, 3]. Brachytherapy is started in the final week(s) of EBRT to ensure an overall treatment duration of less than 50 days. Image-guided adaptive BT based on volumetric imaging (CT/MRI) has improved efficacy and decreased late morbidity after treatment [4]. Nevertheless, treatment with RT has been associated with sexual dysfunction among both GCS and their partners [5-9]. The negative effect of EBRT/BT on sexual functioning is caused by shortening and tightening of the vagina, reduced flexibility, and decreased lubrication, induced by fibrosis and stenosis and mucosal atrophy [10-12]. 
Regular vaginal dilator use after EBRT/BT has been associated with reduced vaginal shortening and/or tightening, although more empirical evidence is needed regarding the effect in reducing sexual problems [13-17]. Regular dilator use has become an essential component of the sexual rehabilitation of GCS worldwide $[16,18,19]$. In spite of this, most patients (75\%) reported being unable to follow dilator use instructions, for example due to being anxious about pain or blood loss, negative emotions regarding dilator use or EBRT/BT, or a lack of support or routine [15, 20-22]. Support during rehabilitation is therefore needed to help GCS experience fewer problems during dilator use and when resuming sexual activity.

An Australian study found that a specific information booklet [23] somewhat increased dilator use 3 and 6 months after treatment compared to "care as usual" (CAU) [24, 25]. Two small Canadian trials compared the effect of two additional psychologist-led group sessions at 1 and 2 months after treatment to CAU $[26,27]$. The intervention group used the dilators more frequently $(65 \%)$ than the CAU group (38\%) at 6 weeks, but no significant difference was found after 6 months (31 versus $19 \%$ ) [26, 27]. The abovementioned interventions, however, found no difference with regard to sexual functioning. The efficacy of sexual rehabilitation interventions may increase by addressing other psychosocial and somatic aspects of sexual functioning possibly affected by cancer treatment, addressing both partners' knowledge and fears, promoting couples' mutual coping to improve sexual health, and including specific sexual therapy techniques $[10$, 28]. Furthermore, it is worthwhile providing follow-up sessions during 1 year after RT to ensure continued support during the recommended 12-month period of dilator use and to investigate whether oncology nurses, who are closely involved with patients during follow-up, would be able to conduct such an intervention after a special training in sexology.

There are no published effective interventions to support GCS with sexual recovery and long-term regular dilator use. Therefore, this prospective, longitudinal, observational pilot study tested a sexual rehabilitation intervention combined with a specific patient information booklet for its clinical feasibility. The intervention was directed at increasing knowledge and offering coping strategies to both patients and their partners with respect to sexual issues after treatment and benefits of dilator use and increasing long-term compliance with dilator use.

\section{Materials and methods}

\section{Participant selection}

In line with experts' recommendations with regard to which patients should be offered support after treatment [18], eligible patients were 18 to 70 years old and had to be treated with primary or postsurgical EBRT/BT for gynecologic cancer. In practice, primary EBRT/BT is mainly given to patients with cervical cancer (primary treatment for International Federation of Obstetrics and Gynecology (FIGO) stages IB2-IIIB) or vaginal cancer (stages I-III) and postsurgical EBRT/BT mainly for cervical cancer (FIGO stage IB1) or endometrial cancer (stage II or III). Women older than 70 were only invited to participate if they were sexually active and wished to resume sexual activity after treatment. Patients with insufficient knowledge of the Dutch language or major psychological problems were excluded and offered counseling by a senior clinical psychologist specialized in sexual rehabilitation. The Leiden University Medical Center Medical Ethics Committee approved the protocol (NL44759.058.13). All participating women provided written informed consent.

\section{The intervention}

A patient information booklet entitled "Sexuality after pelvic radiation for gynecologic cancer: information for women and their partners" was developed. The booklet was developed in collaboration with a multidisciplinary team of sexologists, radiation oncologists, gynecologic oncologists, and oncology nurses. The booklet was subsequently pilot tested by GCS, healthy lower-educated women, and patient advocates from the Dutch gynecologic cancer patient support group "Stichting Olijf". The initial draft of the booklet was partly based on an Australian brochure with permission [23].

A team of clinical psychologist-sexologists developed the intervention based on previous study and intervention results $[18,20,29,30]$. Two senior psychologist-sexologists developed and provided the nurses' training program and monthly 2 -h group supervision. The intervention comprised four faceto-face counseling sessions at 1,2,3, and 6 months after completion of EBRT/BT. An evaluation and closing session was scheduled at 12 months after EBRT/BT. The sessions were planned in line with their radiation oncologist followup visits, except for the session at 2 months. Although not obligatory, the partners of participants in a relationship were invited to join the sessions. The sessions were adapted to the relationship status of participants and the possible partners' presence. During the first session, participants were informed about the intervention, their diagnosis, therapy and possible treatment consequences, and the importance of long-term regular dilator use. Furthermore, participants were recommended to start vaginal dilator use and provided with instructions [18]. A vaginal dilator set, lubricants, and information booklet were provided for free by the participating hospitals.

During the second session, possible barriers to new behaviors such as dilator use, lubricant use, and fear of penetration during dilator use or while resuming sexual activity were discussed. Nurses provided tailored advice depending on the 
participants' situation and reported issues. During the third and fourth sessions, participants' experiences with sexual rehabilitation and dilator use were discussed, and again, tailored advice was given. Couples' mutual coping and support processes were promoted, and specific interventions to address sexual, body image and relationship concerns were included. A follow-up session at 12 months was scheduled to evaluate the course of the sexual rehabilitation in the past year and provide future advice. Furthermore, an extra session could be scheduled between 6 and 12 months after treatment.

\section{The nurses' training}

Four oncology specialist nurses conducted the intervention after receiving 50 hours of skill training. Two senior clinical psychologist-sexologists, with expertise in the conceptualization, methods, and skills, developed and provided the nurses' training program. The training was provided during 6 days, spread over a period of 3 months, and covered the basic principles of sexology, motivational interviewing [31-33], cognitive behavioral interventions $[30,34,35]$, and the treatment protocol itself. The training was delivered using a combination of lecture-style presentation (30\%), role-play and group discussions (35\%), and small-group practice sessions (35\%). Also, guest speakers (a radiation oncologist and gynecologic oncologist) provided education on surgical and radiation treatments, with emphasis on the treatment-related toxicities and management thereof. The nurses received copies of the treatment manual, patient information booklet, and a handbook with the presentations' handouts. During the study period (around 2 years), the nurses received monthly 2-hour group supervision from one of the two clinical psychologist-sexologists.

\section{Measures}

Information was collected from medical records with regard to age, type of cancer, FIGO stage, and hormone replacement therapy (HRT).

\section{Primary outcome measures}

Sexual functioning was measured with the 19-item Female Sexual Function Index (FSFI) total score concerning the subscale sexual desire, arousal, lubrication, orgasm, satisfaction, and genital pain. It was conducted at inclusion to obtain information retrospectively about the participants' situation prior to any diagnosis-related complaints and at 1, 6, and 12 months after RT. Answers are measured with 5- or 6-point Likert scales, and a higher total score (ranging from 2 to 36) indicates better sexual function. The FSFI has been validated among female cancer survivors and showed a good reliability (Cronbach's $\alpha=0.94$ and 0.82 in the current pilot sample) [36].

\section{Secondary outcome measures}

Frequency of vaginal dilatation was assessed monthly using four questions about dilator use frequency, duration, sexual intercourse frequency, and alternative dilator use. Sexually related personal distress was measured with the Female Sexual Distress Scale (FSDS). The FSDS was completed also retrospectively at inclusion and at 1,6 , and 12 months after RT. A higher total score (ranging from 0 to 48 ) indicates more sexually related personal distress [37]. The subscales Anxiety and Depression of the Hospital Anxiety and Depression Scale (HADS) were used to measure anxiety and depression [38]. The subscale Marital (Mal)adjustment of the Maudsley Marital Questionnaire (MMQ) was used to measure relationship dissatisfaction [39]. Higher scores indicate more symptom burden on the three questionnaires. The HADS and MMQ were completed at 1, 6, and 12 months after RT.

\section{Patients' and nurses' exit interviews}

Structured exit interviews were conducted among participants after their last nurse-led consultation at 6 months and before the evaluation session with their nurse at 12 months. They were asked about their experiences with vaginal dilator use, sexual activity, the supportiveness and acceptability of all components of the sexual rehabilitation intervention (including the information booklet), and reasons for discontinuing their participation. The interviews additionally served as a concluding consultation with the researchers about the burden and logistics of the assessments and appointments. Two researchers ( $\mathrm{RB}$ and $\mathrm{MK}$ ) that were not involved in the intervention conducted the interviews. Subsequently, they were verbally transcribed, and the researchers summarized participants' most important evaluations through negotiated consensus.

Exit interviews were also conducted with the nurses regarding the feasibility of the intervention. Furthermore, all nurses' consultations were digitally recorded. Two independent research assistants assessed the nurses' adherence with the treatment protocol and general competency in a sample of 18 out of 85 consults $(21 \%)$.

\section{Statistical methods}

Descriptive statistics were used to evaluate participant characteristics and to assess the nurses' consultations. Nonparametric tests were conducted to analyze the outcome measurements among this small, not normally distributed, pilot sample. Therefore, two-sided, Friedman's ANOVA's and post hoc Wilcoxon signed rank tests for paired samples were used to investigate differences between baseline and the subsequent questionnaires during the study period. Effect sizes for post hoc comparisons were reported as $r$ and classified as small 
( $r=0.1-0.03)$, intermediate $(r=0.3-0.5)$, or strong $(r \geq 0.5)$ [40]. Due to the hypothesis-generating nature of the pilot study, no corrections for multiple testing were applied. A significance level of $5 \%$ was used in all analyses. Analyses were conducted using IBM SPSS version 20 (Armonk, NY, USA).

\section{Results}

\section{Participant characteristics}

Of the 74 patients that received EBRT/BT at 1 of the hospitals during the study period, 34 patients (46\%) were eligible for the study, of whom $31(91 \%)$ were invited and $20(62 \%)$ agreed to participate (see Fig. 1). Participants were between 26 and 71 years of age (mean $(M)=40 \pm 11$ years) and either treated for cervical $(n=18,90 \%)$, vaginal cancer $(n=1,5 \%)$, or endometrial cancer $(n=1,5 \%)$. Fourteen participants were in a partner relationship at the time of inclusion for an average of 15 years $( \pm 13)$. In total, 16 participants used vaginal estriol 3 times weekly during 6 weeks ( 2 to 8 weeks after RT) and 14 participants used HRT (see Table 1).

Four participants stopped participation before the 6-month assessment and another four in the subsequent 6 months (see Fig. 1 and Table 2). Participants had on average 4.5 sessions with their oncology nurse, lasting between 8 and 73 min per session $(M=29 \pm 6 \mathrm{~min})$.

\section{Primary outcome measures}

Participants' sexual functioning significantly changed over time $\left(\chi^{2}(3)=18.00, p<0.001\right)$. Compared to their situation before diagnosis, participants reported lower levels of sexual functioning at 1 month $(p<0.001, r=-0.60)$, as well as at 6 months after RT $(p<0.001, r=-0.59)$. However, after treatment with RT, participants' sexual functioning significantly increased over time. Compared to 1 month after RT, participants' reported higher levels of sexual functioning at 6 months $(p=0.011, r=-0.42)$ and at 12 months after RT $(p=0.012, r=-0.44)$ and continued to improve between 6 and 12 months after RT ( $p=0.015, r=-0.46)$. Their sexual functioning at 12 months was comparable to prior to diagnosis $(p=0.346, r=-0.17)$ (see Fig. 2 and Online Resource 1).

\section{Secondary outcome measures}

At 6 months, 14 out of 16 participants $(88 \%)$ reported using dilators at least twice a week. At 12 months, 9 out of 12 remaining participants $(75 \%)$ dilated at least twice a week and 11 at least once a week (92\%) (see Fig. 3). Participants performed dilation either by resuming sexual intercourse or using vaginal dilators, while $13(65 \%)$ reported having used other types of dilators at least once, namely, vibrators $(n=6$, $30 \%)$, Vaseline tampons $(n=4,20 \%)$, or fingers $(n=7$, $35 \%)$. At 6 months, partnered participants $(n=11)$ gradually

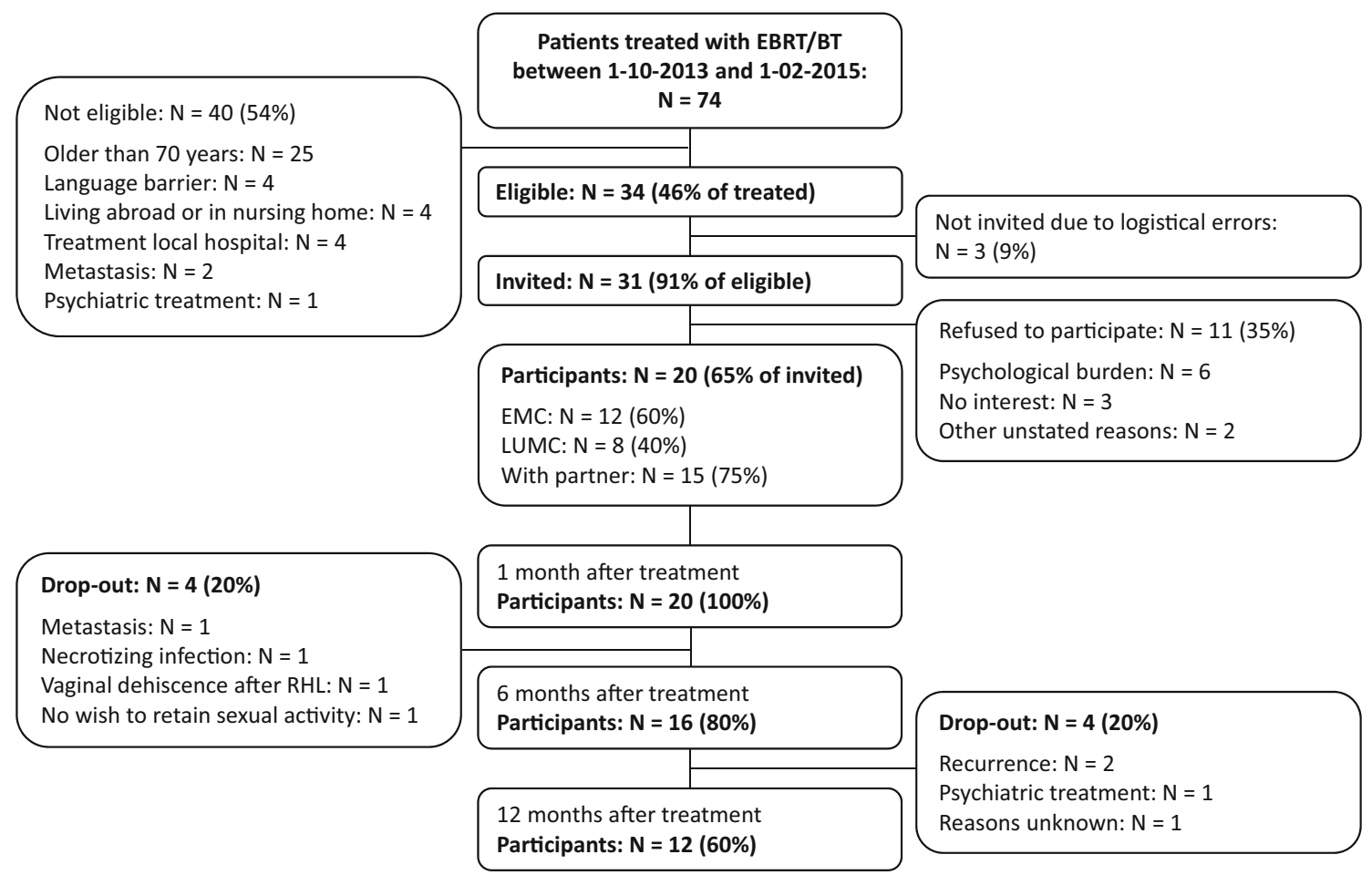

Fig. 1 Flowchart of the participant selection. EMC Erasmus MC Cancer Institute, LUMC Leiden University Medical Center 
Table 1 Cancer treatment and demographic participant characteristics

\begin{tabular}{|c|c|c|c|c|c|c|}
\hline \multirow[b]{2}{*}{ Participant } & \multicolumn{3}{|c|}{ Treatment-related } & \multicolumn{3}{|l|}{ Demographic } \\
\hline & $\begin{array}{l}\text { Cancer type } \\
\text { and FIGO } \\
\text { stage }\end{array}$ & $\begin{array}{l}\text { Primary } \\
\text { treatment }\end{array}$ & $\begin{array}{l}\text { Secondary } \\
\text { treatment }\end{array}$ & HRT & $\begin{array}{l}\text { Age } \\
\text { (years) }\end{array}$ & $\begin{array}{l}\text { Relationship } \\
\text { duration } \\
\text { (years) }\end{array}$ \\
\hline P1 & CC IIB & EBRT/BT & - & Yes & 42 & 21 \\
\hline P2 & CC IIB & EBRT/BT & - & Yes & 32 & 1 \\
\hline P3 & EC IB & RHL & EBRT/BT & No, declined & 40 & - \\
\hline P4 & CC IIB & EBRT/BT & $\mathrm{AH}$ & Yes & 32 & 0 \\
\hline P5 & CC IB1 & EBRT/BT & - & Yes & 43 & - \\
\hline P6 & CC IB2 & EBRT/BT & - & Yes & 31 & - \\
\hline P7 & CC IIB & RHL & EBRT/BT & Yes & 35 & 14 \\
\hline P8 & CC IIB & EBRT/BT & - & Yes & 32 & - \\
\hline P9 & CC IIB & EBRT/BT & - & No, postmenopausal & 57 & 37 \\
\hline P10 & CC IIA & EBRT/BT & - & Yes & 26 & 7 \\
\hline P11 & CC IIB & EBRT/BT & $\mathrm{AH}$ & No, postmenopausal & 51 & 20 \\
\hline P12 & CC IB1 & EBRT/BT & - & No, postmenopausal & 71 & 45 \\
\hline P13 & CC IB2 & EBRT/BT & - & Yes & 43 & - \\
\hline P14 & VC IIB & EBRT/BT & - & Yes & 41 & - \\
\hline P15 & CC IB2 & EBRT/BT & - & Yes & 39 & 7 \\
\hline P16 & CC IIB & EBRT/BT & - & Yes & 31 & 4 \\
\hline P17 & CC IB1 & EBRT/BT & LND/OD & $\begin{array}{l}\text { No, adequate } \\
\text { function of } \\
\text { displaced ovary }\end{array}$ & 26 & 3 \\
\hline P18 & CC IB1 & EBRT/BT & $\mathrm{LND} / \mathrm{OD}$ & Yes & 28 & 11 \\
\hline P19 & CC IIB & EBRT/BT & - & No, declined & 46 & 14 \\
\hline P20 & CC IIB & EBRT/BT & $\mathrm{AH}$ & No, postmenopausal & 50 & 23 \\
\hline
\end{tabular}

FIGO International Federation of Gynecology and Obstetrics, $C C$ cervical cancer, $E C$ endometrial cancer, $V C$ vaginal cancer, $R H L$ radical hysterectomy with pelvic lymphadenectomy, $L N D$ lymph node dissection, $O D$ ovarian displacement, $A H$ abdominal hysterectomy, $H R T$ hormone replacement therapy replaced $(n=2,18 \%)$ or supplemented $(n=6,55 \%)$ vaginal dilator use by having sexual intercourse (see also Online Resource 2).

Participants' sexual distress was not significantly different over time $\left(\chi^{2}(3)=3.67, p=0.299\right)$. Post hoc analyses showed, however, that compared to their pre-diagnosis situation, participants reported higher levels of sexual distress at 1 month $(r=-0.41, p=0.009)$ and 6 months after treatment with RT $(r=-0.42, p=0.012)$ and a trend for higher levels at 12 months after RT $(r=-0.33, p=0.066)$. Also, after treatment with RT, participants' levels of sexual distress did not significantly decrease over time during the intervention. Furthermore, after treatment with RT, participants' levels of depression $\left(\chi^{2}(2)=3.50, p=0.174\right)$, anxiety $\left(\chi^{2}(2)=0.70\right.$, $p=0.704)$, and relationship dissatisfaction $\left(\chi^{2}(2)=3.94\right.$, $p=0.140$ ) did not significantly decrease over time during the intervention. However, post hoc analyses showed that compared to 1-month posttreatment, there were trends for the levels of depression $(r=-0.34, p=0.061)$ and relationship dissatisfaction $(r=-0.33, p=0.091)$ to be lower at 12 months after RT (see Table 3 and Online Resource 1).

\section{Patients' and nurses' concluding remarks}

The exit interviews were conducted with 16 participants and lasted $38 \mathrm{~min}$ on average. They reported that the intervention had been helpful for dilator use and resuming sexual activity $(n=15,94 \%)$. About two thirds mentioned having been bothered by fear of pain or bleeding at first dilator use or sexual activity. However, they also reported that their nurses' support provided reassurance and motivated them to start, which they might not otherwise have done. Furthermore, most women $(n=12,75 \%)$ read the information booklet once or twice and studied the sexual position images more often. Furthermore, according to the participants, having one specific nurse available for extra consultation was important in order to talk comfortably about their personal situation and sexual functioning. Some reported that their partners' presence was not needed, but others considered it important to create mutual understanding.

The nurses reported feeling sufficiently skilled to conduct the intervention and to support participants. From the 
Table 2 Intervention-related participant characteristics

\begin{tabular}{|c|c|c|c|c|}
\hline \multirow[b]{2}{*}{ Participant } & \multicolumn{2}{|l|}{ Intervention-related } & \multicolumn{2}{|l|}{ Drop-out related } \\
\hline & $\begin{array}{l}\text { No. of sessions (by } \\
\text { telephone) }\end{array}$ & $\begin{array}{l}\text { Total } \\
\text { duration } \\
(\mathrm{min})\end{array}$ & $\begin{array}{l}\text { No. of months } \\
\text { after RT }\end{array}$ & Reason \\
\hline P1 & 5 & 112 & - & - \\
\hline P2 & 3 & 150 & 5 & Necrotizing infection \\
\hline P3 & 2 & 42 & 4 & No interest sexual activity \\
\hline P4 & 6 & 304 & - & - \\
\hline P5 & $5(1)$ & 107 & - & - \\
\hline P6 & 5 & 103 & - & - \\
\hline P7 & 6 & 218 & - & - \\
\hline P8 & 5 & 167 & - & - \\
\hline P9 & 5 & 61 & - & - \\
\hline P10 & $6(2)$ & 135 & - & - \\
\hline P11 & 4 & 69 & 5 & $\begin{array}{l}\text { Vaginal dehiscence after surgery for } \\
\text { residual disease }\end{array}$ \\
\hline P12 & 4 & 109 & - & - \\
\hline P13 & 6 & 118 & - & - \\
\hline P14 & 6 & 160 & - & - \\
\hline P15 & 4 & 71 & 9 & Recurrence \\
\hline P16 & 3 & 42 & 6 & Unknown \\
\hline P17 & 4 & 224 & 8 & Psychiatric treatment \\
\hline P18 & 5 & 239 & - & - \\
\hline P19 & 4 & 195 & 8 & Recurrence \\
\hline $\mathrm{P} 20$ & 2 & 101 & 3 & Metastasis (palliative care) \\
\hline
\end{tabular}

consultation recordings, it was apparent that all nurses adhered to the treatment protocol, with flexibility with regard to the specific patient situation. Each nurse demonstrated sufficient competence to conduct the specific interventions.

\section{Discussion}

A nurse-led sexual rehabilitation intervention to help GCSs, treated with EBRT/BT, to initiate vaginal dilator use and address sexual issues, anxieties, and coping problems in the
Fig. 2 Sexual functioning assessed retrospectively about pre-diagnosis and during the intervention at 1,6 , and 12 months after treatment

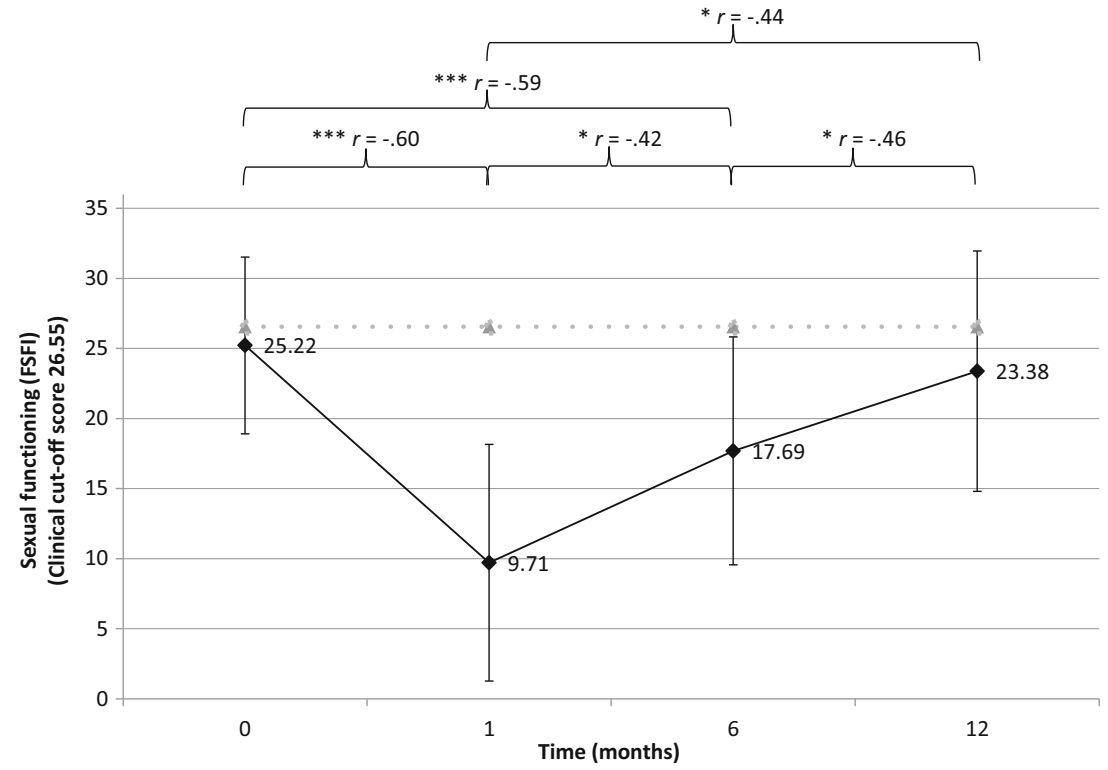


Fig. 3 Average dilation frequency per week $(n=20)$

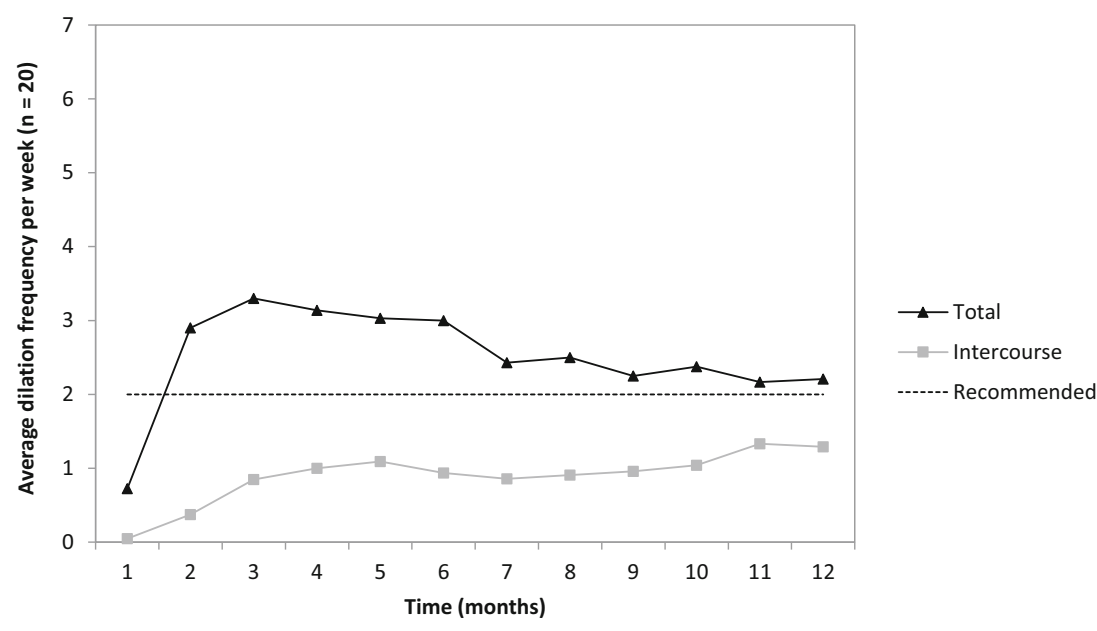

recovery phase was developed, and its feasibility was pilot tested. During the intervention, participants' sexual functioning improved and most participants dilated regularly either by gradually resuming sexual intercourse or by using vaginal dilators or other types of dilation (e.g., vibrator or fingers). Sexual distress, however, continued to be elevated during the 12 months of the intervention. However, as this was a non-randomized feasibility study, a randomized efficacy study should investigate whether the improvements were due to the intervention itself. Most participants reported that the intervention was helpful and the nurses reported having sufficient expertise.

For the first time, the long-term sexual functioning of GCS was studied extensively and was shown to improve and return to participants' reported pre-diagnosis levels, during a 12month sexual rehabilitation intervention [27]. Therefore, it is possible that more follow-up sessions during the first 6 to 12 months after RT and the invitation to the patients' partners to join the sessions lead to better and more sustained sexual recovery among GCS than short individual or peer-group interventions [28].

In contrast to previous studies, a large proportion of this study's participants continued regular long-term dilator use and gradually replaced it with sexual intercourse as well [27]. Jeffries et al. (2006) showed that at 6 months, 4 months after the intervention, only $31 \%$ of the participants dilated at least twice a week [26]. This may be in line with the suggestion that more follow-up sessions may motivate GCS with dilator use and our compliance rate of $88 \%$ is promising. Furthermore, the trend for participants' decreased levels of depression and relationship dissatisfaction at 12 months appeared to indicate better functioning compared to previous GCS cohorts who received no specific support after treatment $[7,41]$. However, participants reported continued sexual distress levels that were comparable with the sexual distress levels reported among a cohort of 72 cervical cancer survivors evaluated 64 months $( \pm 33)$ after EBRT/BT [41]. Our study's participants were evaluated early in the recovery phase, and

Table 3 Outcome measurements completed retrospectively about pre-diagnosis and during the intervention at 1, 6, and 12 months after treatment

\begin{tabular}{|c|c|c|c|c|c|c|c|}
\hline & $\begin{array}{l}0 \text {-month mean } \\
( \pm \mathrm{SD})^{\mathrm{b}} \\
a \\
n=20\end{array}$ & $\begin{array}{l}\text { 1-month mean } \\
( \pm \mathrm{SD}) \\
b \\
n=20\end{array}$ & $\begin{array}{l}\text { 6-month mean } \\
( \pm \mathrm{SD}) \\
c \\
n=16\end{array}$ & $\begin{array}{l}\text { 12-month mean } \\
( \pm \mathrm{SD}) \\
d \\
n=12\end{array}$ & $\chi^{2}$ & $\begin{array}{l}p \\
\text { value }\end{array}$ & $\begin{array}{l}\text { Post hoc } \\
\text { analyses }\end{array}$ \\
\hline \multicolumn{8}{|l|}{ Measure $^{\mathrm{a}}$} \\
\hline Sexual functioning & $25.22(6.31)$ & $9.71(8.44)$ & $17.69(8.13)$ & $23.38(8.58)$ & $18.00 *$ & $<0.001$ & $b<c<d=a$ \\
\hline Sexual distress & $10.25(11.06)$ & $18.25(13.81)$ & $17.94(13.84)$ & $18.91(12.00)$ & 3.67 & 0.299 & $b=c=d>a$ \\
\hline $\begin{array}{l}\text { Relationship } \\
\text { dissatisfaction }\end{array}$ & - & $20.65(30.97)$ & $12.00(8.57)$ & $7.70(5.87)$ & $3.94 * *$ & 0.140 & \\
\hline Anxiety & - & $5.80(4.20)$ & $5.88(3.81)$ & $4.91(3.48)$ & 0.70 & 0.704 & \\
\hline Depression & - & $5.30(3.84)$ & $3.94(3.45)$ & $2.73(3.72)$ & $3.50 * *$ & 0.174 & \\
\hline
\end{tabular}

*Significant difference with $p<0.05 ; * *$ post hoc analyses showed a trend for $b=c>d$ with $p<0.10$

${ }^{a}$ Sexual functioning Female Sexual Functioning Index, sexual distress Female Sexual Distress Scale, anxiety and depression Hospital Anxiety and Depression Scale Subscales Anxiety and Depression, relationship dissatisfaction Maudsley Marital Questionnaire

${ }^{\mathrm{b}} S D$ standard deviation 
therefore, it should be noted that sexual distress among GCS may further recover between 12 and 24 months [9]. How much improvement can be achieved after a sexual rehabilitation intervention compared with care as usual in the long term is unknown.

Although the results obtained in this intervention study are promising, several comments can be made for future reference. Given the uncontrolled nature of this pilot study, it can be argued that the improvement in sexual functioning to pre-diagnosis levels was due merely to the passage of time and that the extra contact with professionals may also improve sexual problems of GCS [9]. In addition, despite the clinical evidence that vaginal dilatation is associated with reduced vaginal complaints and better sexual rehabilitation, firm evidence for its effectiveness is still lacking. Furthermore, a possible selection bias may have occurred since $55 \%$ (6 out of 11) of the patients who declined felt that participation would be a psychological burden. Therefore, the current participants may have experienced less distress or better coping mechanisms. Lastly, this study's dropout rate of $40 \%$ was higher than the $20 \%$ that was expected based on previous intervention studies [26, 27]. However, dropout was only due to disease-related issues. Also, the recruitment of 10 to 15 participants is considered adequate for the purpose of a pilot study [42].

Based on our pilot findings, this study's intervention has proven to be feasible and promising and may improve support for GCS during sexual recovery and vaginal dilatation after EBRT/BT. We are now one step closer to improving sexual health-related care for EBRT/BT survivors. To follow up on participants' improvement during the current nurse-led sexual rehabilitation intervention, long-term efficacy should be investigated in a larger controlled study. Therefore, to evaluate its (cost-)effectiveness, a multicenter, randomized trial with a control group receiving standard care will be initiated.

Acknowledgments This study was funded by the Dutch Cancer Society and Alpe d'Huzes foundation (UL2011-5245).

\section{Compliance with ethical standards}

Conflict of interest The authors declare that they have no conflict of interest.

Open Access This article is distributed under the terms of the Creative Commons Attribution-NonCommercial 4.0 International License (http:// creativecommons.org/licenses/by-nc/4.0/), which permits any noncommercial use, distribution, and reproduction in any medium, provided you give appropriate credit to the original author(s) and the source, provide a link to the Creative Commons license, and indicate if changes were made.

\section{References}

1. Dutch Cancer Registry (2015) Vereniging van Integrale Kankercentra. Available at http://www.cijfersoverkanker.nl. Last accessed 21st of March 2016

2. Waggoner SE (2003) Cervical cancer. Lancet 361:2217-2225. doi:10.1016/S0140-6736(03)13778-6

3. Vale C (2008) Reducing uncertainties about the effects of chemoradiotherapy for cervical cancer: a systematic review and metaanalysis of individual patient data from 18 randomized trials. J Clin Oncol 26:5802-5812. doi:10.1200/JCO.2008.16.4368

4. Rijkmans EC, Nout RA, Rutten IHHM, Ketelaars M, Neelis KJ, Laman MS, Coen VIMA, Gaarenstroom KN, Kroep JR, Creutzberg CL (2014) Improved survival of patients with cervical cancer treated with image-guided brachytherapy compared with conventional brachytherapy. Gynecol Oncol 135:231-238. doi:10.1016/j. ygyno.2014.08.027

5. Gilbert E, Ussher JM, Perz J (2011) Sexuality after gynaecological cancer: a review of the material, intrapsychic, and discursive aspects of treatment on women's sexual-wellbeing. Maturitas 70: 42-57. doi:10.1016/j.maturitas.2011.06.013

6. Jensen PT, Froeding LP (2015) Pelvic radiotherapy and sexual function in women. Transl Androl Urol 4:186-205. doi:10.3978/j. issn.2223-4683.2015.04.06

7. Kirchheiner K, Potter R, Tanderup K, Lindegaard JC, Haie-Meder C, Petric P, Mahantshetty U, Jurgenliemk-Schulz IM, Rai B, Cooper R, Dorr W, Nout RA (2016) Health-related quality of life in locally advanced cervical cancer patients after definitive chemoradiation therapy including image guided adaptive brachytherapy: an analysis from the EMBRACE study. Int J Radiat Oncol Biol Phys 94:1088-1098. doi:10.1016/j.ijrobp.2015.12.363

8. Le Borgne G, Mercier M, Woronoff AS, Guizard AV, Abeilard E, Caravati-Jouvenceaux A, Klein D, Velten M, Joly F (2013) Quality of life in long-term cervical cancer survivors: a population-based study. Gynecol Oncol 129:222-228. doi:10.1016/j. ygyno.2012.12.033

9. Pieterse QD, Kenter GG, Maas CP, de Kroon CD, Creutzberg CL, Trimbos JBM, ter Kuile MM (2013) Self-reported sexual, bowel and bladder function in cervical cancer patients following different treatment modalities: longitudinal prospective cohort study. Int $\mathrm{J}$ Gynecol Cancer 23:1717-1725. doi:10.1097/IGC.0b013e3182a80 a65

10. Bakker RM, Kenter GG, Creutzberg CL, Stiggelbout, AM, Derks M, Mingelen W, de Kroon CD, Vermeer WM, ter Kuile, MM. Sexual distress and associated factors among cervical cancer survivors: a cross-sectional multicentre observational study. Submitted for publication in 2016

11. Brand AH, Bull CA, Cakir B (2006) Vaginal stenosis in patients treated with radiotherapy for carcinoma of the cervix. Int J Gynecol Cancer 16:288-293. doi:10.1111/j.1525-1438.2006.00348.x

12. Jensen PT, Groenvold M, Klee MC, Thranov I, Petersen MA, Machin D (2003) Longitudinal study of sexual function and vaginal changes after radiotherapy for cervical cancer. Int J of Rad Oncol Biol Phys 56:937-949. doi:10.1016/S0360-3016(03)00362-6

13. Decruze SB, Guthrie D, Magnani R (1999) Prevention of vaginal stenosis in patients following vaginal brachytherapy. Clin Oncol 11: 46-48

14. Gondi V, Bentzen SM, Sklenar KL, Dunn EF, Petereit DG, Tannehill SP, Straub M, Bradley KA (2012) Severe late toxicities following concomitant chemoradiotherapy compared to radiotherapy alone in cervical cancer: an inter-era analysis. Int J Radiat Oncol Biol Phys 84:973-982. doi:10.1016/j.ijrobp.2012.01.064

15. Law E, Kelvin JF, Thom B, Riedel E, Tom A, Carter J, Alektiar KM, Goodman KA (2015) Prospective study of vaginal dilator use 
adherence and efficacy following radiotherapy. Radiother Oncol 116:149-155. doi:10.1016/j.radonc.2015.06.018

16. Miles T, Johnson N (2014) Vaginal dilator therapy for women receiving pelvic radiotherapy. Cochrane Database Syst Rev 9: CD007291. doi:10.1002/14651858.CD007291.pub3

17. Velaskar SM, Martha R, Mahantashetty U, Badakare JS, Shrivastava SK (2007) Use of indigenous vaginal dilator in radiation induced vaginal stenosis. Indian J Occup Ther 39:3-6

18. Bakker RM, ter Kuile MM, Vermeer WM, Nout RA, Mens JW, van Doorn LC, de Kroon CD, Hompus WC, Braat C, Creutzberg CL (2014) Sexual rehabilitation after pelvic radiotherapy and vaginal dilator use: consensus using the Delphi method. Int J Gynecol Cancer 24:1499-1506. doi:10.1097/IGC.0000000000000253

19. Clinical Guideline group. International Guidelines on Vaginal Dilation after Pelvic Radiotherapy. http://www.ncsi.org.uk/wpcontent/uploads/Inter-Best-Practice-Guide-Vaginal-Dilators-July2012.pdf. 2012. Oxon, Owen Mumford

20. Bakker RM, Vermeer WM, Creutzberg CL, Mens JW, Nout RA, ter Kuile MM (2015) Qualitative accounts of patients' determinants of vaginal dilator use after pelvic radiotherapy. J Sex Med 12:764773. doi: $10.1111 /$ jsm. 12776

21. Brand AH, Do V, Stenlake A (2012) Can an educational intervention improve compliance with vaginal dilator use in patients treated with radiation for a gynecological malignancy? Int J Gynecol Cancer 22:897-904. doi:10.1097/IGC.0b013e31824d7243

22. Friedman LC, Abdallah R, Schluchter M, Panneerselvam A, Kunos A (2011) Adherence to vaginal dilation following high dose rate brachytherapy for endometrial cancer. Int J Radiat Oncol Biol Phys 80:751-757. doi:10.1016/j.ijrobp.2010.02.058

23. Juraskova I, Lubotzky F (2015) Recovering after pelvic radiation therapy: a guide for women Available from: http://www. targetingcancer.com.au/wp-content/uploads/2015/10/Recoveringafter-Pelvic-Radiation-Therapy-a-guide-for-women.pdf

24. Lubotzky F (2014) The development, pilot and randomised controlled trial of a psychosexual rehabilitation information booklet for women undergoing pelvic radiation therapy for gynaecological or anorectal cancer. Online published $\mathrm{PhD}$ thesis available from: http://ses.library.usyd.edu.au/handle/2123/12819

25. Lubotzky F, Butow P, Nattress K, Hunt C, Carroll S, Comensoli A, Philp S, Juraskova I (2015) Facilitating psychosexual adjustment for women undergoing pelvic radiotherapy: pilot of a novel patient psychoeducational resource. Health Expect:1-12. doi:10.1111/hex.12424

26. Jeffries SA, Robinson JW, Craighead PS, Keats MR (2006) An effective group psychoeducational intervention for improving compliance with vaginal dilation: a randomized controlled trial. Int J Rad Oncol Biol Phys 65:404 411. doi:10.1016/j.jirobp.2005.12.009

27. Robinson JW, Faris PD, Scott CB (1999) Psychoeducational group increases vaginal dilation for younger women and reduces sexual fears for women of all ages with gynecological carcinoma treated with radiotherapy. Int J Radiat Oncol Biol Phys 44:497-506. doi:10.1016/S0360-3016(99)00048-6
28. Scott JL, Kayser K (2009) A review of couple-based interventions for enhancing women's sexual adjustment and body image after cancer. Cancer 15:48-56. doi:10.1097/PPO.0b013e31819585df

29. Weijenborg PTM, ter Kuile MM (2000) The effect of a group programme on women with the Mayer-Rokitansky-Kuster-Hauser syndrome. BJOG 107:365-368. doi:10.1111/j.1471-0528.2000. tb13232.x

30. ter Kuile MM, Weijenborg PTM (2006) A cognitive-behavioral group program for women with vulvar vestibulitis syndrome (VVS): factors associated with treatment success. J Sex Marital Ther 32:199-213. doi:10.1080/00926230600575306

31. Miller WR, Rollnick S (2012) Motivational interviewing: helping people change, 3rd ed. Guilford Press. ISBN 978-1-60918-227-4

32. Palacio A, Garay D, Langer B, Taylor J, Wood BA, Tamariz L (2016) Motivational interviewing improves medication adherence: a systematic review and meta-analysis. J Gen Intern Med 31:929 940. doi:10.1007/s11606-016-3685-3

33. Madson MB, Loignon AC, Lane C (2009) Training in motivational interviewing: a systematic review. J Subst Abus Treat 36:101-109. doi:10.1016/j.jsat.2008.05.005

34. Ter Kuile MM, Melles RJ, de Groot HE, Tuijnman-Raasveld CC, van Lankveld JJDM (2013) Therapist-aided exposure for women with lifelong vaginismus: a randomized waiting-list control trial of efficacy. J Consult Clin Psych 81:1127-1136. doi:10.1037/a0034292

35. van Lankveld JJDM, ter Kuile MM, de Groot HE, Melles R, Nefs J, Zandbergen M (2006) Cognitive-behavioral therapy for women with lifelong vaginismus: a randomized waiting-list controlled trial of efficacy. J Consult Clin Psych 74:168-178. doi:10.1037/0022006X.74.1.168

36. Baser RE, Li Y, Carter J (2012) Psychometric validation of the Female Sexual Function Index (FSFI) in cancer survivors. Cancer 118:4606-4618. doi:10.1002/cncr.26739

37. Derogatis LR, Rosen R, Leiblum S, Burnett A, Heiman J (2002) The Female Sexual Distress Scale (FSDS): initial validation of a standardized scale for assessment of sexually related personal distress in women. J Sex Marital Ther 28:317-330. doi:10.1080/00926230290001448

38. Spinhoven P, Ormel J, Sloekers PP, Kempen GI, Speckens AE, Van Hemert AM (1997) A validation study of the Hospital Anxiety and Depression Scale (HADS) in different groups of Dutch subjects. Psychol Med 27:363-370. doi:10.1017/S0033291796004382

39. Arrindell WA, Schaap C (1985) The Maudsley Marital Questionnaire (MMQ): an extension of its construct validity. Br J Psychiatry 147:295-299. doi:10.1192/bjp.147.3.295

40. Cohen J (1988) Statistical power analysis for the behavioral sciences (2nd ed.). Lawrence Erlbaum Associates, Hillsdale, NJ

41. Vermeer WM, Bakker RM, Kenter GG, de Kroon CD, Stiggelbout AM, ter Kuile MM (2015) Sexual issues among cervical cancer survivors: how can we help women seek help? Psychooncology 24:458-464. doi:10.1002/pon.3663

42. Hertzog MA (2008) Considerations in determining sample size for pilot studies. Res Nurs Health 31:180-191. doi:10.1002/nur.20247 